\title{
A brighter future in the fight against hepatitis
}

\author{
Public health and research efforts directed at managing and targeting viral hepatitis have borne fruit in recent \\ decades. However, more work is necessary to meet the goals of preventing transmission and treating infection to \\ eliminate the enormous burden of hepatitis worldwide.
}

1 n 2012, the World Health Organization (WHO) established a Global Hepatitis Program with the goal of fully preventing and treating viral hepatitis. This month, the WHO hopes to increase public awareness through the official World Hepatitis Day, on 28 July. In this issue, Nature Medicine features a series of Review and Perspective articles that discuss promising research and clinical efforts and continuing challenges in viral hepatitis.

Hepatitis B virus (HBV) and HCV are primarily responsible for the high global prevalence of hepatitis disease and for the morbidity and mortality associated with chronic infection. A key challenge for the management of hepatitis is its silent progression, as acute hepatic failure rarely occurs. Infection is often asymptomatic, causing liver scarring and damage decades later in up to $30 \%$ of people infected with $\mathrm{HCV}$, and the proportion is even higher in those infected with $\mathrm{HBV}$ at birth or during early childhood. Inadequate recognition of infection and region-specific variation in prevalence and risk groups hinders diagnosis and precludes timely treatment. The lack of sufficiently widespread antibody screening to identify all exposed individuals and of follow up with RNA testing, a technique not yet available for routine medical use, to distinguish people with active virus, results in incorrect estimates of infection and increased transmission. In the case of hepatitis $\mathrm{C}$, recent human studies showed that less than half of the infected people in the United States knew they carried the virus (Hepatology 55, 1652-1661, 2012), a number that may be higher in areas with limited disease-control tools. Moreover, the harsh side effects of pegylated interferon- $\alpha$ and ribavirin force many infected people to opt out of this standard therapy, contributing to viral persistence in the community and prevalence of chronic disease.

The advent of effective antivirals is changing the therapeutic landscape, and the goal for eradicating hepatitis viruses may not be as distant as it seemed five years ago, high treatment costs notwithstanding. Current antiviral therapies do not cure chronic hepatitis B, which affects about 210 million people worldwide. At the 2013 International Liver Congress, new approaches to eliminate the HBV replication template, which persists inside liver cells, by modulating host processes such as epigenetic mechanisms and hepatocyte regeneration showed promise and may offer the potential of a cure in the future. For HCV, which affects about 150 million individuals worldwide, there have been rapid advances in drugs. Two protease inhibitors approved in 2011 greatly improved responses in patients infected with the predominant genotype 1; however, host genetic variability affecting antiviral efficacy, evolving drug resistance and the lack of coverage to inhibit all existing HCV genotypes are major drawbacks. Also, these new drugs must be given with the standard therapy, which exacerbates side effects. Second-generation antivirals with different viral targets are under development, and combination strategies should improve efficacy and may even eradicate the virus. Although these therapies are promising, resistance may still develop, and monitoring the emergence of resistant variants will be necessary for guiding treatment choices.

An interferon-free therapy for hepatitis $\mathrm{C}$ may also soon exist. In April, a triple combination of direct-acting antivirals without interferon showed efficacy in treatment-naive individuals and in nonresponders to standard of care. And in May, four clinical trials tested an inhibitor of viral polymerase, sofosbuvir, in patients infected with HCV (N. Engl. J. Med. 368, 1867-1887, 2013). In combination with ribavirin, sofosbuvir showed increased efficacy against genotypes 2 and 3 compared to both standard of care and placebo, and adding pegylated interferon alpha-2a to the combination achieved broad genotype coverage. Patients with unacceptable side effects to standard therapy or who were unresponsive to previous therapies may therefore benefit from these new approaches. Research on host factors required for the HCV life cycle has also yielded targets that may overcome virus-acquired resistance and circumvent side effects. A recent example is the targeting of microRNA-122, which is liver specific and necessary for viral replication (N. Engl. J. Med. 368, 1685-1694,2013) Although long-term studies are necessary to address their safety and toxicity in the long run, interferon-free strategies may become the future of hepatitis $\mathrm{C}$ therapy.

But the holy grail for eradicating and decreasing the burden of any infectious disease is a prophylactic vaccine. Prevention of infection with the effective $\mathrm{HBV}$ vaccines and with improved medical and lifestyle practices has reached impressive levels in developed countries, and continuing efforts to improve testing and implement mass vaccination programs in low-income countries should achieve similar results in these regions. A working vaccine for $\mathrm{HCV}$, however, still remains elusive, in part because of the lack of experimental systems to study the virus and the lack of animal models to test vaccine candidates. Moreover, because this virus has developed mechanisms of persistence and has an enormous genetic diversity, vaccines will need to induce both neutralizing antibodies and T cell-mediated responses to achieve broad, lasting cross-protection. Unraveling how the host immune response clears the virus during the course of natural infection and prevents persistence will help us understand what constitutes protective immunity and provide a rationale to develop an effective pan-genotype vaccine.

The goals of preventing infection, slowing disease progression and curing chronic hepatitis will undoubtedly require continuing research and clinical efforts. Pharmaceutical companies should be encouraged to keep investigating future compounds to overcome the existing therapeutic barriers, and public awareness efforts should be intensified to underscore to funding and public health agencies that, although we are closer, we are still far from achieving the goals proposed to tackle these silent elusive killers. 